\title{
Clinical Effects of Korean Red Ginseng on Attention Deficit Hyperactivity Disorder in Children: An Observational Study
}

\author{
Suk Hwa Lee ${ }^{1}$, Woo Sung Park², and Myung Ho Lim ${ }^{1^{*}}$ \\ ${ }^{1}$ Department of Psychiatry and Environmental Health Center, Dankook University, College of Medicine, \\ Cheonan 330-715, Korea \\ ${ }^{2}$ Department of Pediatrics, Dankook University, College of Medicine, Cheonan 330-715, Korea
}

We investigated the clinical effects of Korean red ginseng on attention deficit hyperactivity disorder (ADHD) in children. Eighteen subjects aged between 6 and 14 diagnosed with ADHD based on the Diagnostic and Statistical Manual of Mental Disorders, fourth edition diagnosis criteria were enrolled. Korean red ginseng (Panax ginseng) at 1,000 mg b.i.d. was administered to the subjects for 8 weeks. Eighteen children completed the questionnaire and clinical assessment by visiting the hospital at baseline, then in the first, fourth and eighth weeks. Clinical assessments were performed by using the ADHD Diagnostic System (ADS, a computerized attention assessment program), the abbreviated Conners' rating scale in addition to psychiatric interviews. After 8 weeks, significant differences were found in the omisssion errors of ADS ( $78.56 \pm 43.33$ at baseline, $55.17 \pm 21.44$ at 8 weeks, $p<0.023)$, Conners ADHD Rating Scale $(13.78 \pm 6.32$ at baseline, $9.50 \pm 4.80$ at 8 weeks, $p<0.042)$ and Spielberger State Anxiety Scale (30.94 \pm 6.25 at baseline, $28.83 \pm 6.23$ at 8 weeks, $p<0.024)$. In the Korean Personality Inventory for Children, a significant reduction of score was found in the physical development scale (56.44 \pm 9.63 at baseline, $50.94 \pm 8.91$ at 8 weeks, $p<0.017)$ and social dysfunction scale $(56.33 \pm 6.82$ at baseline, $51.94 \pm 7.13$ at 8 weeks, $p<0.025)$. These results suggest that Korean red ginseng may be effective in improving inattentiveness in ADHD children, but it remains uncertain if it improves the general severity of ADHD, depression, anxiety personality and behavioral changes.

Keywords: Panax ginseng, Attention deficit hyperactivity disorder (ADHD), Korean red ginseng, Child

\section{INTRODUCTION}

Attention deficit hyperactivity disorder (ADHD) is a common child psychiatric disorder characterized by the cognitive problems such as attention deficit, and the behavioral problems such as hyperactivity and impulsivity. ADHD is a very common disorder with a $2 \%$ to $7.6 \%$ prevalence among children of school age in Korea $[1,2]$. Genetic factor is considered the strongest among the causes of ADHD. However, it is recently considered that the etiology may be more complex, as genetics fac- tors are combined with environmental factors [3,4]. The complex action of dopamine and noradrenaline has been continuously reported as the pathophysiologcal cause of ADHD [4]. In addition, it has recently been reported that dopamine level was reduced in the synapse of prefrontal cortex related to continuous attention in ADHD [4]. Agents most frequently used for the treatment of ADHD include methylphenidate and dextroamphetamine, atomoxetine. Efficacy can be seen in $75 \%$ of these agents [4]. (c) This is an Open Access article distributed under the terms of the Creative Commons Attribution Non-Commercial License (http://creativecommons.org/licenses/by-nc/3.0/) which permits unrestricted non-commercial use, distribution, and reproduction in any medium, provided the original work is properly cited.
Received 14 Jan. 2011, Revised 01 Mar. 2011, Accepted 01 Mar. 2011

*Corresponding author

E-mail: paperose@dku.edu

Tel: +82-10-2640-1498, Fax: +82-41-561-3007 
Efficacy is very high compared to the efficacy of agents for the treatment of other neuropsychiatric disorders, which may be because all of these agents have impact on dopamine or norepinephrine, the neurotransmitters in the neuronal synapse, resulted in positive therapeutic effects on ADHD patients [4].

Recently, complementary therapeutic methods have been introduced in other countries to supplement drug treatment, which is the new therapeutic intervention for ADHD [5]. Other therapeutic intervention includes herbal medicines affecting dopamine metabolism, such as Hypericum perforatum (St. John's wort) [5], medicines that improve circulation, such as Ginkgo biloba [6], and ginseng [7].

Saponin, phenol, polyacetylene, alkaloid, polysaccaride are known to be the main physiologically active components of ginseng. Its effects on strengthening physique and augmenting hypertension, on fatigue and stress-related diseases are already known [8,9]. In addition, results of previous pre-clinical studies suggest that ginseng have significant impact on neuroendocrine function, carbohydrate and lipid metabolism, provides antioxidizing action to the cardiovascular system, modulates immune and cardiovascular functions, and exerts other effects on the central nervous system [10].

Ginseng has been shown to exert nootrophic effect on the central nervous system that improves learning, memory, attention, sensory-motor performance and cognitive processing [11,12]. Furthermore, it has been reported from a pre-clinical study that ginsenosides, a ginseng constituent, increased dopamine and norepinephrine concentrations in the cerebral cortex [13]. These properties may explain the effects of ginseng extracts in improving attention, cognitive function, sensory-motor function and auditory reaction time [14]. These effects suggest that long-term administration of ginseng extract may result in positive growth in the neurodevelopment of an immature brain. Third, ginseng components are also reported to have neuroprotective properties [15]. It has been reported that ginseng extracts, saponin, $20(\mathrm{~S})-\mathrm{Rg}_{3}$ and $\mathrm{Rc}$, protect brain tissue by repressing nitric oxide (NO) generation in the central nervous system and cerebrovascular endothelial cells [16]. Ginsenoside $\mathrm{Rb}_{1}$ and $\mathrm{Rg}_{3}$ exert protective effect by suppressing damage on the neurons via inhibiting NO generation associated with glutamate-induced neurotoxicity [17].

Although various pre-clinical studies were conducted on ginseng to investigate its effects on the central nervous system, few clinical studies have been conducted. Until recently, there have only been a few reports on the clini- cal effects of Korean red ginseng on psychiatric disorders in Korea. Even though there is a report on the effect of co-administration of American ginseng and G. biloba on ADHD [15], no study has been conducted in Korean and beyond on the efficacy of ginseng monotherapy.

The aim of this study was to investigate the effect of Korean red ginseng (Panax ginseng) to improve the symptoms of ADHD.

\section{MATERIALS AND METHODS}

\section{Subjects and materials}

This study was single arm open case study (Fig. 1). Study subjects were comprised of 18 children aged between 6 and 14 who visited the department of child psychiatry at Dankook University Hospital from August to September 2010 diagnosed with ADHD based on psychiatric interview conducted by child psychiatrists and the Diagnostic and Statistical Manual of Mental Disorders, fourth edition (DSM-IV) diagnosis criteria [18]. They had no history of psychiatric drug administration for ADHD and no abnormality was found on medical and physical examination. Detailed explanation and consultation were provided to the caregivers and patient children who participated in the study about the purpose of the Korean red ginseng (capsules of Korean red ginseng powder) manufactured by Korea Ginseng Corporation study and informed consents were received from them. This study was approved by the Institutional Review Board of Dankook University Hospital. We provided sufficient information regarding the expectable advantages and side effects of Korean red ginseng administration.

\section{Methods}

At the first visit, basic history was evaluated and physical examination was performed on each subject who visited the Department of Psychiatry as outpatient. On the day of the first visit, clinical scales, such as the Korean version of Child Behavior Checklist (K-CBCL) [19], Korean Personality Inventory for Children (KPI-C) [20,21], Kovac's Children's Depression Inventory (CDI) [22], State Anxiety Inventory (SAIC) and Trait Anxiety Inventory (TAIC) [23] were used by the child psychiatrist to perform assessments on the children. In addition, we performed attention deficit hyperactivity disorder Diagnostic System (ADS) [24] which is widely used in clinics to diagnose ADHD and assess therapeutic effects as the computerized tool designed to assess the continuous performance shown in the children whose chief complaint is $\mathrm{ADHD}$. We also performed the questionnaire survey 


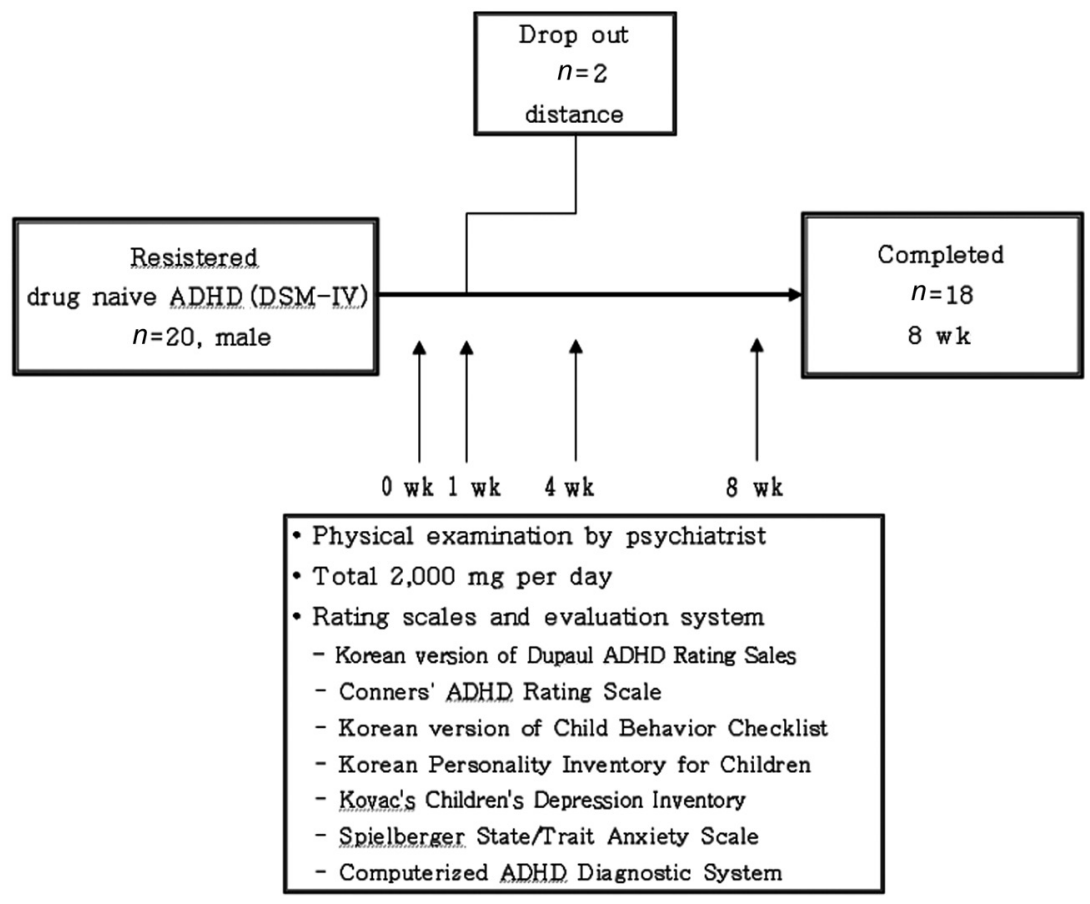

Fig. 1. Schematic of the experimental design. Eighteen children with attention deficit hyperactivity disorder (ADHD) received Korean red ginseng at $1,000 \mathrm{mg}$ b.i.d. for 8 weeks period. Physical examination and clinical rating scales by psychiatrist were performed at baseline, 1 week, 4 weeks, 8 weeks for each treatment. DSM-IV, Diagnostic and Statistical Manual of Mental Disorders, fourth edition.

with the children's parents and carried out Abbreviated Conners Parent-Teacher Rating Scale-Revised [25] and Korean version of Dupaul Attention Deficit Hyperactivity Disorder Rating Sales (K-ARS) [26].

At the second visit, patients commenced Korean red ginseng therapy. Patients were administered capsules of Korean red ginseng powder produced by the Korean Tobacco and Ginseng Corporation, and is registered as a health product. Each capsule is comprised of Korean red ginseng extract $82.15 \%$ and miscellaneous gelatin materials $17.85 \%$. The Korean red ginseng extract content of one capsule included $500 \mathrm{mg}$ of Korean red ginseng. The daily quantity corresponded to $2 / 3(2,000 \mathrm{mg})$ of the daily recommendation for adults $(3,000 \mathrm{mg})$. Each subject was administered half a tablet twice daily, irrespective of age and weight, for 8 weeks without dose adjustment.

Follow-up visits were scheduled one, four and eight weeks after the initial consultation. The same clinical ratings were repeatedly carried out at each visit to evaluate the efficacy of Korean red ginseng. In addition, ADHD symptoms and side effects were assessed through interview with the children and their caregivers at each visit. The capsule of Korean red ginseng included red ginseng extract $82.15 \%$, miscellaneous gelatin materials $17.85 \%$. The Korean red ginseng extract of one capsule included the amount of $20 \mathrm{mg}$ per gram of red ginseng.

\section{Assessment tools \\ Epidemiological questionnaire}

The history questionnaire contains questions on sex, age, school grade, medical and developmental history.

\section{Computerized Attention Deficit-Hyperactivity Disorder Diagnostic System}

ADS is a computerized program being widely used to diagnose ADHD and assess the continuous performance function in children with attention-deficit or hyperactivity. As one of the most popular Continuous Performance Tests in Korea, ADS was developed and standardized by Shin et al. [24]. It has two components: visual and auditory. ADS assesses general attention ability and its detailed evaluation index includes omission errors, commission errors, hit reaction time mean, hit reaction time standard deviation, attentiveness, and risk taking.

\section{Abbreviated Conners Parent-Teacher Rating Scale (re- vised)}

Goyette, Conners, and Ulrich replicated and designed a 10-item index from the Conners' Parent-Teacher Rating Scale, which was initially developed by Conners in 
1970 to measure major symptoms of childhood ADHD. In Korea, Oh [25] reported its reliability from a study of domestic children.

\section{Korean version of Dupaul Attention Deficit Hyperactivity Disorder Rating Sales}

Developed by Dupaul based on the DSM-IV diagnostic criteria for ADHD, the Dupaul ADHD Rating Scales is used for assessment by parent and teacher on child behavior. The scales, with 18 items, is proven for its validity in differentiating children with ADHD from those without, and designed to effectively differentiating three subtypes of ADHD: predominantly inattentive, predominantly hyperactive-impulsive, and combined hyperactiveimpulsive and inattentive. Korean standardization has been achieved by So et al. [26]. and children with ADHD may score above 19 by parents or 18 by teachers.

\section{Kovac's Children's Depression Inventory}

This CDI was developed by Kovacs to measure the degree of children depression by modifying the Beck's depression inventory for children aged between 6-14 [22]. Cho and Lee classified scores over 22 as mild depressive state, over 26 as medium depressive state, and over 29 as severe depressive state [27].

\section{Spielberger State-Trait Anxiety Inventory}

This is a useful assessment tool to measure anxiety clinically. It is useful in identify clinically anxious group and psychiatric patients and it also has the advantage to measure the anxious state of normal adults without psychiatric disorder. It was developed by Spielberger et al. [23]. and translated into Korean by Kim [28], and the reliability and validity were studied. In the case of trait anxiety inventory scale, the scores 39-42 indicate a little high trait anxiety, scores 43-46 indicate a considerably high trait anxiety, and scores 47 or higher indicate very high trait anxiety [29]. In the case of state anxiety inventory scale, the scores 41-44 indicate a little high state anxiety, scores 45-48 indicate a considerably high state anxiety, and scores 49 or higher indicate very high state anxiety [29].

\section{The Korean version of Child Behavior Checklist}

The K-CBCL rating scale used in this study was based on Child Behavior Checklist developed by Achenbach. It was translated into Korean by $\mathrm{Oh}$ and Lee [19]. This scale is for children at the age between 4 and 18 and its problem behavior syndrome scale consists of three internalizing problems (social withdrawal, somatic com- plaints, depression/anxiety), two externalizing problems (delinquent behavior, aggressiveness), social problems, thought problems and attention problems.

\section{Korean Personality Inventory for Children}

$\mathrm{KPI}-\mathrm{C}$, another rating scale used in this study, is useful in measuring the psychological characteristics of children's emotion, behavior and cognition. KPI-C was prepared by Wirt et al. [20]. They standardized the Personality Inventory for Children developed by Wirt et al. [20] for Korean children. The subjects of KPI-C are children and adolescents aged between 4 and 15, and KPI-C is supposed to be documented by parents or caregiver who have lived with the children or adolescents for last six months. It contains a total of 255 yes-or-no questions. A total of 16 scales including four validity scales, self-resilience scale and 11 clinical scales for verbal development, physical development, anxiety, depression, somatization, delinquency, hyperactivity, family relations, social relations, psychoticism and autism.

\section{Analytical method}

We performed the repeated measures $t$-test to compare the results before the Korean red ginseng medicine administration and one, four and eight weeks after the administration through the Conners ADHD scale, K-ARS, Kovac's CDI, SAIC, TAIC, K-CBCL, KPI-C and ADS. SPSS ver. 12.0 (SPSS Inc., Chicago, IL, USA) was used for the statistical analysis and the significance level was set to the p-value less than 0.05 .

\section{RESULTS}

\section{Subjects}

Eighteen out of 20 male patients completed the required tests and scales at 4 follow-up visits during the eight-wk period. However, two were unable to attend for follow-up visits after the first visit and were thus excluded from the statistical analysis, which began from the first visit. Three of the 18 patients were taking escitalpram $5 \mathrm{mg}$ before study entry, and the dose remained unchanged throughout the study.

Among patients who completed all follow-up visits, two experienced bad taste of the Korean red ginseng capsule. They elected to open the capsules and dissolve the content in water before swallowing the mixture. No significant gastro-intestinal side effects were reported, except a degree of repulsive feeling experienced by patients due to the unique flavor of red ginseng. 
Table 1. Rating scales and diagnostic system of ADHD ( $n=18)$

\begin{tabular}{lcccccc}
\hline \multicolumn{1}{c}{ Rating scales } & Baseline & $1 \mathrm{wk}$ & $4 \mathrm{wk}$ & $8 \mathrm{wk}$ & $\mathrm{F}$ & $p$-value \\
\hline Attention Deficit-Hyperactivity Disorder Diagnostic System & & & & & \\
$\quad$ Omission error & $78.56 \pm 43.33$ & $74.00 \pm 41.17$ & $63.11 \pm 28.47$ & $55.17 \pm 21.44$ & 6.30 & 0.023 \\
$\quad$ Commission error & $75.00 \pm 25.31$ & $70.22 \pm 31.35$ & $70.17 \pm 35.17$ & $58.83 \pm 30.23$ & 0.030 & 0.872 \\
$\quad$ Response time & $47.67 \pm 15.76$ & $51.39 \pm 15.76$ & $47.06 \pm 11.65$ & $47.11 \pm 15.65$ & 0.38 & 0.544 \\
$\quad$ RTSD & $88.28 \pm 42.57$ & $88.33 \pm 41.75$ & $68.39 \pm 26.62$ & $58.44 \pm 14.84$ & 0.07 & 0.789 \\
Conners ADHD Scale & $13.78 \pm 6.32$ & $12.06 \pm 5.58$ & $10.50 \pm 5.64$ & $9.50 \pm 4.80$ & 4.89 & 0.042 \\
Dupaul ADHD Scale & & & & & \\
$\quad$ Total & $23.22 \pm 10.56$ & $19.00 \pm 9.62$ & $18.17 \pm 10.49$ & $14.11 \pm 6.85$ & 4.19 & 0.057 \\
$\quad$ Attention & $13.00 \pm 5.37$ & $10.72 \pm 5.03$ & $10.39 \pm 5.28$ & $8.72 \pm 4.04$ & 3.52 & 0.079 \\
$\quad$ Hyperactivity & $10.22 \pm 5.86$ & $8.28 \pm 5.37$ & $7.83 \pm 5.45$ & $5.39 \pm 3.76$ & 4.02 & 0.062 \\
Learning Disorder Scale & $4.28 \pm 4.07$ & $2.28 \pm 2.85$ & $2.17 \pm 2.85$ & $2.61 \pm 3.70$ & 2.66 & 0.123 \\
Kovac Depression Scale & $8.89 \pm 6.30$ & $8.28 \pm 5.23$ & $8.83 \pm 6.03$ & $6.39 \pm 4.42$ & 0.68 & 0.422 \\
Spielberger State Anxiety Scale & $30.94 \pm 6.25$ & $29.89 \pm 6.58$ & $30.00 \pm 6.44$ & $28.83 \pm 6.23$ & 6.21 & 0.024 \\
Spielberger Trait Anxiety Scale & $30.06 \pm 7.42$ & $27.22 \pm 5.87$ & $25.94 \pm 4.80$ & $25.00 \pm 5.75$ & 1.76 & 0.203 \\
\hline
\end{tabular}

These data represent mean $\pm S D$, statistical value by repeated measures $t$-test, adjusted age. Significant $p<0.05$.

ADHD, attention deficit hyperactivity disorder; RTSD, standard deviation of response time.

Table 2. Korean version of Child Behavior Checklist of attention deficit hyperactivity disorder $(n=18)$

\begin{tabular}{|c|c|c|c|c|c|c|}
\hline Rating scales & Baseline & $1 \mathrm{wk}$ & $4 \mathrm{wk}$ & $8 \mathrm{wk}$ & $\mathrm{F}$ & $p$-value \\
\hline \multicolumn{7}{|c|}{ Korean version of Child Behavior Checklist } \\
\hline Withdrawn & $56.33 \pm 4.80$ & $53.06 \pm 4.28$ & $52.22 \pm 3.02$ & $51.56 \pm 2.91$ & 1.54 & 0.233 \\
\hline Somatic complaints & $52.61 \pm 4.51$ & $51.44 \pm 3.67$ & $51.39 \pm 3.38$ & $50.39 \pm 1.42$ & 0.48 & 0.498 \\
\hline Anxious/depressed & $53.78 \pm 5.72$ & $53.56 \pm 4.45$ & $51.67 \pm 3.09$ & $51.50 \pm 3.01$ & 2.16 & 0.161 \\
\hline Social problem & $57.22 \pm 5.50$ & $56.61 \pm 6.50$ & $55.94 \pm 5.30$ & $53.50 \pm 4.46$ & 0.02 & 0.893 \\
\hline Thought problems & $55.50 \pm 5.80$ & $53.33 \pm 5.56$ & $52.17 \pm 3.88$ & $51.72 \pm 2.91$ & 0.68 & 0.422 \\
\hline Attention problems & $57.78 \pm 5.58$ & $55.67 \pm 4.47$ & $53.89 \pm 3.95$ & $52.33 \pm 2.89$ & 4.03 & 0.062 \\
\hline Delinquent behavior & $53.50 \pm 4.88$ & $52.61 \pm 4.07$ & $51.22 \pm 2.37$ & $51.28 \pm 3.91$ & 0.93 & 0.350 \\
\hline Aggressive behavior & $56.72 \pm 8.45$ & $56.22 \pm 7.15$ & $53.22 \pm 4.39$ & $52.78 \pm 4.31$ & 3.29 & 0.088 \\
\hline Internalizing problems & $54.28 \pm 4.27$ & $52.61 \pm 3.94$ & $51.22 \pm 2.56$ & $50.78 \pm 1.59$ & 1.55 & 0.232 \\
\hline Externalizing problems & $55.83 \pm 6.96$ & $55.33 \pm 5.93$ & $52.67 \pm 3.69$ & $52.17 \pm 3.93$ & 4.06 & 0.061 \\
\hline Total behavior problems & $54.44 \pm 4.34$ & $54.06 \pm 4.57$ & $51.67 \pm 2.54$ & $51.28 \pm 2.08$ & 4.24 & 0.056 \\
\hline Sex problems & $51.50 \pm 3.45$ & $52.06 \pm 5.13$ & $50.00 \pm 0.00$ & $50.50 \pm 2.12$ & 3.54 & 0.078 \\
\hline Emotional problems & $51.00 \pm 2.40$ & $51.11 \pm 2.30$ & $50.22 \pm 0.65$ & $50.00 \pm 0.00$ & 0.83 & 0.376 \\
\hline
\end{tabular}

These data represent mean $\pm S D$, satistical value by repeated measures $t$-test, adjusted age. Significant $p<0.05$.

\section{Therapeutic effects}

With respect to change in ADHD symptoms observed through ADS, significant difference was found in omission error $(\mathrm{F}=6.30, p=0.023)$ and while no significant difference were others (Table 1). In the Conners ADHD scale, the score was $13.78 \pm 6.32$ before the administration, $12.06 \pm 5.58$ one week later, $10.50 \pm 5.64$ four wk later, and $9.50 \pm 4.80$ eight wk later, showing a significant difference in the score $(\mathrm{F}=4.89, p=0.042)$ (Table 1). In $\mathrm{K}-\mathrm{CBCL}$, a significant score difference was not found in all subscales (Table 2). In KPI-C, a significant score reduction was found in the physical development scale $(\mathrm{F}=7.07, p=0.017)$ and social relations scale $(\mathrm{F}=6.15$, $p=0.025$ ), while no significant difference was found in others (Table 3). 
Table 3. Korean Personality Inventory for Children of attention deficit hyperactivity disorder $(n=18)$

\begin{tabular}{llccccc}
\hline \multicolumn{1}{c}{ Rating scales } & Baseline & $1 \mathrm{wk}$ & $4 \mathrm{wk}$ & 8 wk & F & $\mathrm{p}$-value \\
\hline Korean Personality Inventory for Children & & & & & & \\
$\quad$ Verbal development & $57.44 \pm 11.06$ & $56.00 \pm 9.78$ & $56.06 \pm 10.00$ & $54.22 \pm 11.07$ & 1.07 & 0.316 \\
$\quad$ Physical development & $56.44 \pm 9.63$ & $55.00 \pm 9.79$ & $53.56 \pm 8.15$ & $50.94 \pm 8.91$ & 7.07 & 0.017 \\
Anxiety & $54.61 \pm 10.32$ & $52.72 \pm 10.33$ & $49.00 \pm 9.68$ & $48.06 \pm 9.49$ & 0.07 & 0.793 \\
Depression & $54.83 \pm 9.87$ & $53.50 \pm 12.32$ & $52.44 \pm 9.65$ & $52.78 \pm 12.27$ & 2.13 & 0.163 \\
Somatic concern & $43.39 \pm 10.46$ & $42.00 \pm 12.50$ & $42.17 \pm 11.12$ & $42.00 \pm 11.71$ & 0.00 & 0.949 \\
Delinquency & $54.83 \pm 10.49$ & $55.89 \pm 10.85$ & $52.61 \pm 9.78$ & $54.72 \pm 10.67$ & 0.99 & 0.334 \\
Hyperactivity & $58.28 \pm 8.51$ & $57.50 \pm 11.67$ & $53.78 \pm 10.84$ & $52.89 \pm 11.60$ & 1.23 & 0.283 \\
Family dysfunction & $54.11 \pm 8.63$ & $52.67 \pm 10.20$ & $50.22 \pm 10.94$ & $49.11 \pm 12.16$ & 0.66 & 0.428 \\
Social dysfunction & $56.33 \pm 6.82$ & $54.06 \pm 7.14$ & $52.28 \pm 6.82$ & $51.94 \pm 7.13$ & 6.15 & 0.025 \\
Psychoticism & $49.67 \pm 12.84$ & $48.78 \pm 10.98$ & $48.22 \pm 8.38$ & $43.50 \pm 10.33$ & 0.00 & 0.953 \\
Autism & $53.22 \pm 11.68$ & $51.11 \pm 13.64$ & $50.17 \pm 10.78$ & $47.28 \pm 10.93$ & 2.06 & 0.170 \\
\hline
\end{tabular}

These data represent mean $\pm S D$, statistical value by repeated measures $t$-test, adjusted age.

Significant $p<0.05$.

\section{DISCUSSION}

It has been shown by many pre-clinical studies that ginseng plays the role of modulating neurotransmitters as it stimulates and represses the central nervous system. In more than 20 ginsenosides, $\mathrm{Rb}_{1}$ and $\mathrm{Rg}_{1}$ are known to exert effects on the central nervous system [30,31]. Ginsenoside particularly refers to saponin contained in ginseng out of saponins contained in many plants [32]. Meanwhile, there have been reports on its effect in improving cognitive function in animals and humans. However, no study clearly elucidated the exact mechanism of ginseng's positive effect on cognitive function. However, various hypotheses have been derived through pre-clinical studies. Previous studies conducted in Korea focused on the impact of Korean red ginseng on cognitive function in normal subjects [33].

In the early 1960's, it was reported that ginseng and saponin improved memory in animals with memory impairment and improve physical performance and learning disorder. Subsequently, previous results were validated as various active ginseng components exerted positive impact on brain function in pre-clinical and clinical studies [34]. In addition, some pre-clinical studies reported that hypoglycemia exacerbates cerebral infarction and damages cognitive function, and the blood sugar modulating effect of ginseng improved the cognitive functions in normal volunteering subjects who experienced hypoglycemia after fasting. Furthermore, placebo-control doubleblind studies showed that ginseng improves calculation performance and augments tiredness in subjects after continuous cognitive task performance [35]. These results from pre-clinical studies showed that ginseng saponin exerts effect on the sensitivity of dopamine receptors. Since the known etiological mechanism of ADHD is dopamine and norepinephrine abnormality in the central nervous system, the results may be harmonized with the pre-clinical study results.

Omission errors of ADS measure inattention, one of the representative cognitive function. We assumed that the significant decreasing result of omission errors of ADS in our study may be related to the restoration of impaired cognitive function on ADHD with children. Recently, Einat [36] reported that chronic ginseng had a significant effect on reducing spontaneous locomotor activity as part of behavioral symptoms but not on the distribution of activity and the performance of mice. But the result of our study not found its relationship and hyperactivity, as part of behavioral symptoms of ADHD.

The study by Lyon et al. [15] is the only study regarding the effect of American ginseng on ADHD. This study was a clinical, open-label study with 36 ADHD children at the age between 3 and 17. In this study, American ginseng (Panax quinquefolium) extract $200 \mathrm{mg}$ and $G$. biloba $50 \mathrm{mg}$ were administered for four wk and the result showed that Corners ADHD scale score measured by their parents was significantly improved from the second week of the treatment [15]. In this study, score reduction less than five points in Conners ADHD scale for parents was found in $31 \%$ to $67 \%$ of subjects in the second week of treatment, score reduction less than five points was found in $76 \%$, and score reduction less than ten points 
was found in 53\% in the fourth week of treatment. In addition, only 5 children (15\%) out of 36 complained of minor side effects, including perspiration, headache and fatigue. However, correlation between these symptoms and G. biloba could not be identified because psychostimulants, such as methylphenidate and dexedrine, were administered concurrently. It has also been reported that pre-existing symptoms, such as emotional impulsivity and hyperactivity, became stronger in two children. As discussed previously, the study by Lyon et al. [15], is the only study that investigated the clinical effects of American ginseng extracts and G. biloba in ADHD children. However, since the two agents were administered in parallel, above all, in this study as an open clinical study, the effect of ginseng alone could not be elucidated. Moreover, because the parents' self-assessment scale was only used for the assessment of ADHD symptoms, these symptoms could not be considered to be objectively assessed. Also $39 \%$ of subjects were administered psychostimulant drug. In our study, a significant difference was found only in the omission error of ADS, which is different from the result of Lyon et al. [15] where a significant difference was found both in the inattention and hyperactive-impulsivity of DSM-IV. There may be various factors to the different results and one of them is that the single effect of ginseng was difficult to be evaluated in the study of Lyon et al. [15] because Ginko extract was mixed with American ginseng for the administration.

In our study, on the other hand, although there were 3 patient children who were taking different medicine (escitalopram $5 \mathrm{mg} /$ day), there was an advantage in assessing the single effect of Korean red ginseng because none of psychostimulants was used. In addition, for the assessment of ADHD symptoms, we not only applied the self-report type rating scale but also carried out objective evaluation of continuous competency by means of ADS. Thus, our study can be considered objective. Considering that a statistically significant score change was found in omission error $(\mathrm{F}=6.30, p=0.023)$ and the Conners ADHD scale ( $\mathrm{F}=4.89, p=0.042)$ in our study, a positive result was found as in the previous study of Lyon et al. [15].

On the other hand, ADHD is known to be associated with many coexisting symptoms. Depression and anxiety are symptoms associated with ADHD in more than $50 \%$ of cases [37]. In particular, it has been reported that gensenoside $\mathrm{Rb}_{1}, \mathrm{Rg}_{1}, \mathrm{Rg}_{5}$ and $\mathrm{Rk}$ mixture reduces hyperactivity in mice [38]. In one study the effect of gensenoside $\mathrm{Rb}_{1}, \mathrm{Rg}_{1}$ and $\mathrm{Rg}_{5}$, and $\mathrm{Rk}$ mixture was reported to be smaller than that of diazepam, with superior side-effect profile.

A statistically significant score change was found in SAIC ( $\mathrm{F}=6.21, p=0.024)$. But it did not translate into clinical significance, because the mean baseline score was 30.94 , lower than the threshold score for anxiety disorder. More studies are required to investigate the effect of ginseng on ADHD in addition to anxiety and mood disorder in the future (Table 3).

The limitations of our study are as follows: first, this is a small-scale open-label case study with 18 ADHD children enrolled without controls, the clinical responses to Korean red ginseng found in this study cannot be generalized for the entire ADHD population. The placebo effect might effect the result of this study because of the lack of control group. The calculation revealed that the sample size of 30 subjects is required to obtain the power that is $90 \%$ or higher in the four times of repeated measures $t$-test with the patients group. Our study was conducted with 18 subjects and the power was $67.4 \%$. This indicates that the therapeutic effect of Korean red ginseng on ADHD cannot be sufficiently accounted for by the result in this study. It also indicates that the result from the clinical inventory that showed no therapeutic effect might have been false negative. Thus, we anticipate a large-scale study with a larger number of patients and controls in the future. However, we performed the power program analysis for the four sessions of repeated measures of $t$-test with 18 subjects and the result showed that the effect size was 0.33 . As the editor recommended; second, since red ginseng solid powder solely derived from Korean red ginseng was used, this study did not elucidate the specific constituent of ginseng that improved symptoms; third, the effective dose might not have been administered to patients, because a single dose was applied irrespective of patient height and weight. In generally, an infant whose weight is $30 \mathrm{~kg}$ or over is given the dose that is similar to that for an adult clinically. The dose in this study was 2,000 mg of Korean red ginseng, which is $2 / 3$ of the general dose for an adult, 3,000 mg. In determining the dose, we considered that the mean weight of the infants at the age between 6 and 14, the subjects of this study, was 22 to $53 \mathrm{~kg}$ in Korea. It would have been better to set up different administration doses per body weight referring to the age; fourth, since laboratory tests were not performed at study entry and only physical examination was conducted, other conditions which could potentially influence study outcome were not evaluated; fifth, the study was only conducted in male patients and the outcomes may not be applicable to female ADHD patients; sixth, the long-term efficacy of ginseng was not 
assessed, since the study was conducted over a short period of time.

Further studies should be conducted in the future to clarify the clinical effect of ginseng on ADHD by augmenting the various limitations of this study. Ginseng has the potential to be used complementary therapy for ADHD in addition to conventional drug treatment, if its efficacy and safety are validated in the future [39].

\section{ACKNOWLEDGEMENTS}

This study was supported by the grant (2010) from the Korean Society of Ginseng funded by Korean Ginseng Corporation.

\section{REFERENCES}

1. Cho SC, Shin YO. Prevalence of disruptive behavior disorders. J Korean Acad Child Adolesc Psychiatry 1994; 5:141-149.

2. Pyo KS, Park SH, Kim SH, Cho YR, Kim HR, Moon KR. The prevalence of attention deficit hyperactivity disorder in urban elementary school children. Gwangju: Department of Psychiatry, Josun University, 2001.

3. Eaves LJ, Silberg JL, Meyer JM, Maes HH, Simonoff E, Pickles A, Rutter M, Neale MC, Reynolds CA, Erikson MT et al. Genetics and developmental psychopathology: 2. The main effects of genes and environment on behavioral problems in the Virginia Twin Study of Adolescent Behavioral Development. J Child Psychol Psychiatry 1997;38:965-980.

4. Biederman J, Faraone SV. Attention-deficit hyperactivity disorder. Lancet 2005;366:237-248.

5. Butterweck V, Wall A, Lieflander-Wulf U, Winterhoff $H$, Nahrstedt A. Effects of the total extract and fractions of Hypericum perforatum in animal assays for antidepressant activity. Pharmacopsychiatry 1997;30 Suppl 2:117124.

6. Ramassamy C, Naudin B, Christen Y, Clostre F, Costentin J. Prevention by Ginkgo biloba extract (EGb 761) and trolox $\mathrm{C}$ of the decrease in synaptosomal dopamine or serotonin uptake following incubation. Biochem Pharmacol 1992;44:2395-2401.

7. Ha DC, Ryu GH. Chemical components of red, white and extruded root ginseng. J Korean Soc Food Sci Nutr 2005;34:247-254.

8. Park CK, Jeon BS, Yang JW. The chemical components of Korean ginseng. Food Ind Nutr 2003;8:10-23.

9. Bhattacharya SK, Muruganandam AV. Adaptogenic activity of Withania somnifera: an experimental study using a rat model of chronic stress. Pharmacol Biochem Behav 2003;75:547-555.

10. Gillis CN. Panax ginseng pharmacology: a nitric oxide link? Biochem Pharmacol 1997;54:1-8.

11. Benishin $\mathrm{CG}$. Actions of ginsenoside $\mathrm{Rb}_{1}$ on choline uptake in central cholinergic nerve endings. Neurochem Int 1992;21:1-5.

12. Sloley BD, Pang PK, Huang BH, Ba F, Li FL, Benishin CG, Greenshaw AJ, Shan JJ. American ginseng extract reduces scopolamine-induced amnesia in a spatial learning task. J Psychiatry Neurosci 1999;24:442-452.

13. Itoh T, Zang YF, Murai S, Saito H. Effects of Panax ginseng root on the vertical and horizontal motor activities and on brain monoamine-related substances in mice. Planta Med 1989;55:429-433.

14. D’Angelo L, Grimaldi R, Caravaggi M, Marcoli M, Perucca E, Lecchini S, Frigo GM, Crema A. A double-blind, placebo-controlled clinical study on the effect of a standardized ginseng extract on psychomotor performance in healthy volunteers. J Ethnopharmacol 1986;16:15-22.

15. Lyon MR, Cline JC, Totosy de Zepetnek J, Shan JJ, Pang P, Benishin C. Effect of the herbal extract combination Panax quinquefolium and Ginkgo biloba on attentiondeficit hyperactivity disorder: a pilot study. J Psychiatry Neurosci 2001;26:221-228.

16. Kim DH, Jung JS, Suh HW, Huh SO, Min SK, Son BK, Park JH, Kim ND, Kim YH, Song DK. Inhibition of stress-induced plasma corticosterone levels by ginsenosides in mice: involvement of nitric oxide. Neuroreport 1998;9:2261-2264.

17. Chen X, Salwinski S, Lee TJ. Extracts of Ginkgo biloba and ginsenosides exert cerebral vasorelaxation via a nitric oxide pathway. Clin Exp Pharmacol Physiol 1997;24:958-959.

18. American Psychiatric Association. Diagnostic and statistical manual of mental disorders: DSM-IV. 4th ed. Washington, DC: American Psychiatric Association, 1994.

19. Oh KJ, Lee H. Development of Korean child behavior checklist: a preliminary study. J Korean Neuropsychiatr Assoc 1990;29:452-462.

20. Wirt RD, Lachar D, Klinedenst JK. Multidimensional description of child personality: a manual for the personality inventory for children. Los Angeles: Western Psychological Services; 1977.

21. Hong CH, Kim ST, Kim JH, Hwang ST. Development and standardization of the Korean Personality Inventory for Children. Ment Health Res 2001;20:198-212.

22. Kovacs M. The children's depression inventory: a selfrated depression scale for school-aged youngsters [unpublished manuscript]. Pittsburgh: University of Pittsburgh, 
1983.

23. Spielberger CD, Gorsuch RL, Lushene RE. STAI manual for the state-trait anxiety inventory. Pal Alto: Consulting Psychologist Press; 1970.

24. Shin MS, Cho SC, Chun SY, Hong KE. A study of the development and standardization of ADHD diagnostic system. J Korean Acad Child Adolesc Psychiatry 2000;11:91-99.

25. Oh KJ. Assessment of children with attention deficit hyperactivity disorder. J Korean Acad Child Adolesc Psychiatry 1990;1:65-76.

26. So YK, Noh JS, Kim YS, Ko SG, Koh YJ. The reliability and validity of Korean parent and teacher ADHD rating scale. Korean J Neuropsychiatr Assoc 2002;41:283-289.

27. Cho SC, Lee YS. Development of the Korean form of the Kovacs' children's depression inventory. Korean J Neuropsychiatr Assoc 1990;29:943-956.

28. Kim JT. Relationship between trait anxiety and sociability: focused on STAI of Spielberger [dissertation]. Seoul: Graduate School of Korea University, 1978.

29. Cho SC, Choi JS. Development of the Korean form of the state-trait anxiety inventory for children. Seoul J Psychiatry $1989 ; 14: 150-157$.

30. Liu M, Zhang JT. Influence of ginsenoside $\mathrm{Rb}_{1}$ and $\mathrm{Rg} 1$ on some central neurotransmitter receptors and protein biosynthesis in mouse brain. Acta Pharmacol Sin 1996;17:171-174.

31. Chen XC, Chen Y, Zhu YG, Fang F, Chen LM. Protective effect of ginsenoside Rg1 against MPTP-induced apop- tosis in mouse substantia nigra neurons. Acta Pharmacol Sin 2002;23:829-834.

32. Lian XY, Zhang Z, Stringer JL. Protective effects of ginseng components in a rodent model of neurodegeneration. Ann Neurol 2005;57:642-648.

33. Park CU. Effect of Panax ginseng on CNS. J Ginseng Res 2001;25:11-18.

34. Ma Y, Eun JS, Oh KW. Therapeutic effects of ginseng on psychotic disorders. J Ginseng Res 2007;31:117-126.

35. Reay JL, Kennedy DO, Scholey AB. Effects of Panax ginseng, consumed with and without glucose, on blood glucose levels and cognitive performance during sustained 'mentally demanding' tasks. J Psychopharmacol 2006;20:771-781.

36. Einat H. Chronic oral administration of ginseng extract results in behavioral change but has no effects in mice models of affective and anxiety disorders. Phytother Res 2007;21:62-66.

37. Hong KE, Kim JH, Shin MS, Ahn DH. Diagnostic classification and assessment of psychiatrically referred children with inattention or hyperactivity. J Korean Acad Child Adolesc Psychiatry 1996;7:190-202.

38. Carr MN, Bekku N, Yoshimura H. Identification of anxiolytic ingredients in ginseng root using the elevated plusmaze test in mice. Eur J Pharmacol 2006;531:160-165.

39. Rucklidge JJ, Johnstone J, Kaplan BJ. Nutrient supplementation approaches in the treatment of ADHD. Expert Rev Neurother 2009;9:461-476. 